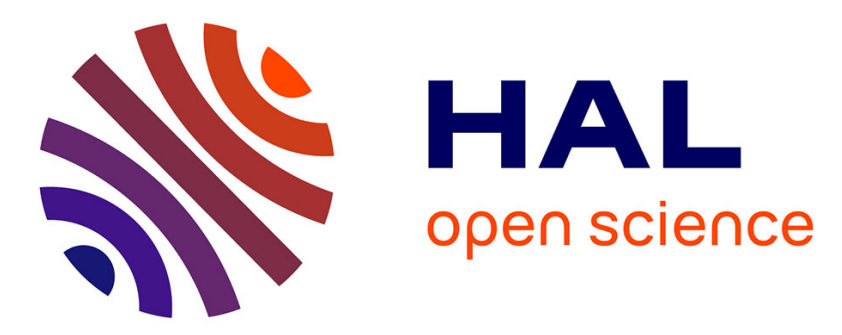

\title{
State tracking control for Takagi-Sugeno models
}

Souad Bezzaoucha, Benoît Marx, Didier Maquin, José Ragot

\section{To cite this version:}

Souad Bezzaoucha, Benoît Marx, Didier Maquin, José Ragot. State tracking control for TakagiSugeno models. IEEE Multi-Conference on Systems and Control, MSC 2014, Oct 2014, Antibes, France. 10.1109/CCA.2014.6981613 . hal-01023620

\section{HAL Id: hal-01023620 https://hal.science/hal-01023620}

Submitted on 25 Nov 2021

HAL is a multi-disciplinary open access archive for the deposit and dissemination of scientific research documents, whether they are published or not. The documents may come from teaching and research institutions in France or abroad, or from public or private research centers.
L'archive ouverte pluridisciplinaire HAL, est destinée au dépôt et à la diffusion de documents scientifiques de niveau recherche, publiés ou non, émanant des établissements d'enseignement et de recherche français ou étrangers, des laboratoires publics ou privés. 


\title{
State tracking control for Takagi-Sugeno models
}

\author{
Souad Bezzaoucha ${ }^{1}$, Benoît Marx ${ }^{2,3}$, Didier Maquin ${ }^{2,3}$ and José Ragot ${ }^{2,3 *}$ \\ $\ddagger$
}

\begin{abstract}
This work addresses the model reference tracking control problem. It aims to highlight the encoutered difficulties and the proposed solutions to achieve the tracking objective for nonlinear systems described by Takagi-Sugeno (T-S) models.

Different control strategies are exposed. Exact state tracking is proposed and structural conditions for it are given. Approximate state tracking is also studied. The choice of the reference model to be tracked is discussed, as well as the criterion to be minimized to achieve given tracking objectives.
\end{abstract}

\section{Introduction and paper outline}

The main objective of this work is to deal with reference model tracking for nonlinear systems described by Takagi-Sugeno (T-S) models. The T-S models are known to be an efficient way to deal with the problems of estimation and control of nonlinear systems by writing them in a polytopic form. Originally introduced by [1], the T-S representation allows to exactly describe nonlinear systems, provided that the nonlinearities are bounded. This is reasonable since state variables as well as parameters of physical systems are bounded, and so is the input of the system which may be considered naturally stable, or equipped with a stabilizing control (see for example [2] and the references therein).

Despite an abundant literature on stability conditions of T-S models, few authors have dealt with the tracking problem for all the state of the system; indeed most of the works deal with the tracking of the system output, which generally reduces to some combinations of these variables. One can refer to some works concerned with

\footnotetext{
*1 University of Bordeaux, IMS lab-CNRS, Automatic control group, 351 cours de la liberation, 33405 Talence, France. e-mail : souad. bezzaoucha@ims-bordeaux. fr

$\dagger 2$ Université de Lorraine, CRAN, UMR 7039, 2 avenue de la Forêt de Haye, Vandoeuvre-lès-Nancy Cedex, 54516, France

$\ddagger 3$ CNRS, CRAN, UMR 7039, France. e-mails:benoit.marx, didier.maquin, jose.ragot auniv-lorraine.fr
}

state or output feedback with $H_{\infty}$ performances [3], [4] and [5]. The nonlinear tracking control problem is expressed in terms of Linear Matrix Inequality (LMI) and is based on the T-S and Parallel Distributed Compensation (PDC) structures (an $\mathscr{L}_{2}$ tracking performance related to the tracking error is formulated and a PDC state feedback control (or output feedback control) is designed. See [5], [4] for examples). However, in the cited references, a referred "suitable" choice for the reference model is made without any explanations nor details.

The last remark motivated the present study. In fact, either for the linear case or the nonlinear one, few works detail the influence of the reference model choice, which is not a trivial task. In [6] for example, the authors referred to the Erzberger's conditions, but with no further explanations. For these reasons, in the proposed work, a focus is made not only on the design control procedure, but also on the tracking (matching) conditions.

The paper is organized as follows. In section II, the structural conditions to achieve state tracking are introduced in the T-S case. These conditions are an extension of the well known Erzberger's conditions. Two procedures for state tracking are considered: for the first one, the controller structure is firstly fixed and then the structural conditions and the appropriate gains of the controller are deduced. For the second one, no prerequisited control law is considered, with the objective to achieve a null tracking error for both strategies. In section III, the quadratic optimal control with an extension to the Model Predictive Control for T-S models are presented. Simulation examples are given in section IV. Finally, section V summarizes the obtained results.

\section{Structural conditions for exact state tracking for T-S systems}

\subsection{Model and objective}

Let us consider the following T-S model [1]:

$$
x_{k+1}=A_{k} x_{k}+B_{k} u_{k}
$$


s.t. $x_{k} \in R^{n_{x}}$ and $u_{k} \in R^{n_{u}}$ with:

$$
A_{k}=\sum_{i=1}^{r} \mu_{i, k}\left(\xi_{k}\right) A_{i}, \quad B_{k}=\sum_{i=1}^{r} \mu_{i, k}\left(\xi_{k}\right) B_{i}
$$

where $B_{k}$ is supposed to be a full column rank matrix and where the weighting functions $\mu_{i, k}\left(\xi_{k}\right)$ depend on the so-called premise variable $\xi_{k}$ which may be a state, input, or output combination. These weighting functions satisfy the following convex sum property:

$$
0 \leq \mu_{i, k}\left(\xi_{k}\right) \leq 1, \sum_{i=1}^{r} \mu_{i, k}\left(\xi_{k}\right)=1
$$

The considered linear reference model is the following:

$$
x_{r, k+1}=A_{r} x_{r, k}+B_{r} u_{r, k}
$$

s.t. $x_{r, k} \in R^{n_{x}}, u_{r, k} \in R^{n_{u}}$ and where the desired performances are defined by the choice of the matrices $A_{r}$ and $B_{r}$.

The ideal tracking objective is to adjust, at each instant $k$, the control $u_{k}$ in such a way that the system state $x_{k}$ follows the reference model state $x_{r, k}$ with a null tracking error. For this purpose, two procedures may be considered. The first strategy consists in setting the controller structure and then deduce the appropriate structural conditions that must satisfy the reference model and controller gains. The second strategy is not based on a prerequisited control law structure. The idea is to find, as for the first strategy, the appropriate structural conditions, but also an analytical expression for the control law. If the ideal tracking is not reachable, some compromises need to be defined such as, for example, tracking of a subset of the states, instead of all of them.

\subsection{Prerequisited control law}

In order to achieve the tracking objective, the following control law is considered:

$$
u_{k}=K_{k} x_{k}+K_{r, k} u_{r, k}
$$

Substituting (5) into (1), the closed-loop system is:

$$
x_{k+1}=\left(A_{k}+B_{k} K_{k}\right) x_{k}+B_{k} K_{r, k} u_{r, k}
$$

The matching conditions for the reference model and the system are then obtained by comparing the closedloop system (6) and the reference model (4). They are given by:

$$
\left\{\begin{array}{c}
A_{k}+B_{k} K_{k}=A_{r} \\
B_{k} K_{r, k}=B_{r}
\end{array}\right.
$$

From (7), in order to have a solution in respect to the gain $K_{k}$ and $K_{r, k}$, the following rank conditions have to be fullfiled:

$$
\left\{\begin{array}{rlc}
\operatorname{rank}\left[B_{k}\right] & =\operatorname{rank}\left[B_{k} \mid A_{r}-A_{k}\right] \\
\operatorname{rank}\left[B_{k}\right] & =\operatorname{rank}\left[B_{k} \mid B_{r}\right]
\end{array}\right.
$$

where $B_{k}$ and $A_{k}$ are defined in (2). If conditions (8) are fulfilled, then at each sampling time, the gains $K_{k}$ and $K_{r, k}$ are given by:

$$
K_{r, k}=B_{k}^{+} B_{r}, \quad K_{k}=B_{k}^{+}\left(A_{r}-A_{k}\right)
$$

with $B_{k}^{+}$a suitable pseudo-inverse matrix of the full column rank $B_{k}$ matrix.

Note that in order to satisfy the matching conditions (8), from definitions (2), since the system matrices $A_{k}$ and $B_{k}$ depend on the time, one sufficient, but not unique, condition is to consider the matrices $A_{i}, B_{i}$ and $A_{r}, B_{r}$ in the following canonical form:

$$
\begin{gathered}
A_{i}=\left(\frac{A_{0}}{\bar{A}_{i}}\right), A_{r}=\left(\frac{A_{0}}{\bar{A}_{r}}\right) \\
B_{i}=\left(\frac{0_{n_{x}-n_{u}}}{b_{i}}\right), B_{r}=\left(\frac{0_{n_{x}-n_{u}}}{b_{r}}\right)
\end{gathered}
$$

with $A_{0}$ a matrix of dimension $\left(n_{x}-n_{u}\right) \times n_{x}, \bar{A}_{i}$ and $\bar{A}_{r}$ matrices of dimensions $n_{u} \times n_{x} . b_{i}$ and $b_{r}$ are of dimension $n_{u} \times n_{u}$. The structure (10) means that:

1. the $\left(n_{x}-n_{u}\right)$ first rows of the matrices $A_{i}$ are equal to the $\left(n_{x}-n_{u}\right)$ first rows of the matrix $A_{r}$

2. the $\left(n_{x}-n_{u}\right)$ first rows of the matrices $B_{i}$ are null

3. the $\left(n_{x}-n_{u}\right)$ first rows of the matrix $B_{r}$ are null

allowing to fully satisfy the rank conditions (8). It is important to note that the matching conditions (8) between the reference model and the system depend on the choice of the control law, $u_{k}$ given by (5). It means that these conditions have to be adapted when changing the structure of the control law.

\subsection{Numerical example}

To illustrate the above conditions, let us consider the following academic example:

$$
\begin{gathered}
A_{r}=\left(\begin{array}{ccc}
0.2 & 0.5 & 0 \\
-0.2 & 0.99 & -0.1 \\
0 & 0 & 0.2
\end{array}\right), B_{r}=\left(\begin{array}{l}
0 \\
0 \\
1
\end{array}\right) \\
A_{1}=\left(\begin{array}{ccc}
0.2 & 0.5 & 0 \\
-0.2 & 0.99 & -0.1 \\
0 & 0 & 0.1
\end{array}\right), B_{1}=\left(\begin{array}{c}
0 \\
0 \\
1.5
\end{array}\right)
\end{gathered}
$$




$$
A_{2}=\left(\begin{array}{ccc}
0.2 & 0.5 & 0 \\
-0.2 & 0.99 & -0.1 \\
0 & 0 & 1.1
\end{array}\right), B_{2}=\left(\begin{array}{c}
0 \\
0 \\
-0.5
\end{array}\right)
$$

The weighting functions are taken as:

$$
\mu_{1, k}=\frac{2-\sin \left(x_{1, k}\right)-\tanh \left(x_{2, k}\right)}{4}, \mu_{2, k}=1-\mu_{1, k}
$$

Applying the tracking control law (5) with (9), the system and model reference states are depicted in figure 1 (respectively noted $x_{i}$ and $x_{i r}, i=1, \ldots, 3$ ). In figure 2 , the control inputs $u_{r, k}$ and $u_{k}$ are represented.
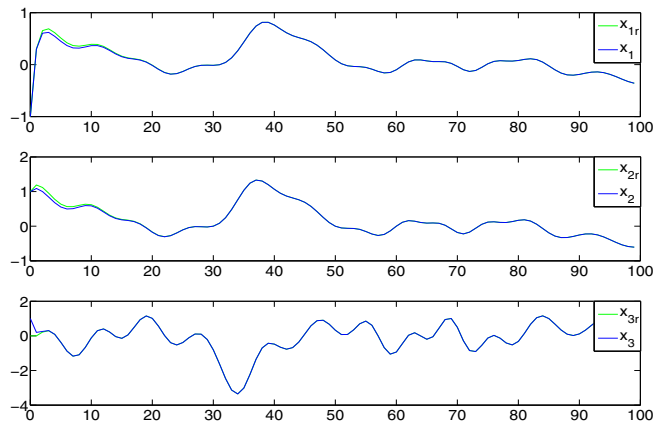

Figure 1. System and model reference states
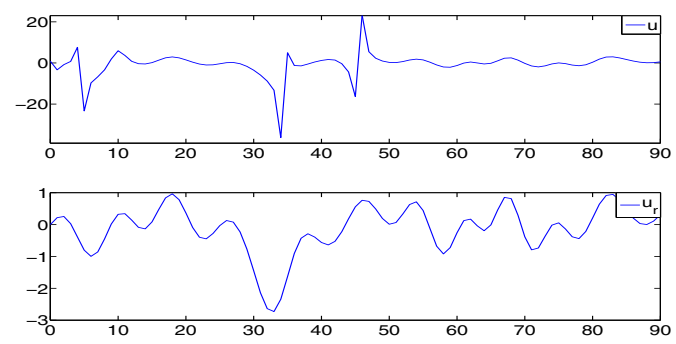

Figure 2. Control inputs $u_{r, k}$ and $u_{k}$

From the depicted figures, one can see that the control tracking is efficient for all the three states under the specified structural conditions.

\subsection{No prerequisited control law}

In this subsection, no prerequisited structure of the control law is considered. Then, at time $k$, in order to achieve the tracking objective at time $k+1$ with a null state tracking error between (4) and (1), meaning:

$$
x_{r, k+1}=A_{k} x_{k}+B_{k} u_{k}
$$

the control law $u_{k}$ has to verify:

$$
B_{k} u_{k}=x_{r, k+1}-A_{k} x_{k}
$$

Note that the exact state tracking is ensured, i.e. (13) has a solution in respect to $u_{k}$, only if the following matching condition is fulfilled at each sampling time:

$$
\operatorname{rank}\left[B_{k}\right]=\operatorname{rank}\left[B_{k} \mid x_{r, k+1}-A_{k} x_{k}\right]
$$

The tracking control is deduced and given by the following equation:

$$
u_{k}=\left(B_{k}^{T} B_{k}\right)^{-1} B_{k}^{T}\left(x_{r, k+1}-A_{k} x_{k}\right)
$$

and the reader is invited to compare the two structures (16) and (5) with the help of (9).

Remark 1 If the premise variables $\xi_{k}$ of the weighting functions $\mu_{i, k}$ depend on the input $u_{k}$, the control law (16) will be implicit (i.e. $u_{k}=F\left(u_{k}\right)$ ) since $A_{k}$ and $B_{k}$ are input depending (2). A solution may be given by an iterative algorithm with the following recurrence:

$$
u_{k}^{(j+1)}=\left(\left(B_{k}^{(j)}\right)^{T} B_{k}^{(j)}\right)^{-1}\left(B_{k}^{(j)}\right)^{T}\left(x_{r, k+1}-A_{k}^{(j)} x_{k}\right)
$$

with $B_{k}^{(j)}=\sum_{i=1}^{r} \mu_{i, k}\left(u_{k}^{(j)}\right) B_{i}, A_{k}^{(j)}=\sum_{i=1}^{r} \mu_{i, k}\left(u_{k}^{(j)}\right) A_{i}, j=$ $0, \ldots, N$ with $N$ the number of iterations and $u_{k}^{0}$ the input initialization (may be taken as $u_{r, k}$ for example).

The convergence of this algorithm may be proved locally, but it will not be considered in the present work since it is not the purpose of the study (see [7], [8], [9] for more details).

As mentioned in the beginning of this section, to achieve the exact tracking, the system and model reference must be in a certain canonical form (structural conditions). Depending on the reference and system models, these conditions may be impossible to meet and thus must be relaxed. One way is to apply the tracking to some states only, but it can affect the functioning of the system since other states are left free. A better way is to use an approximate tracking of all the states.

\section{Approximate state tracking for T-S sys- tems}

In this section, the optimized tracking using the norm of the tracking error is addressed. This part concerns the optimal control with the introduction of the MPC for T-S models. As it was mentioned above, due to the restrictiveness of the exact tracking structural conditions, one can consider another approach to relax these 
structural conditions. This approach is the so-called quadratic optimal control for T-S models and aims at minimizing the tracking error. This is a natural way to deal with the problem by optimizing the tracking error. Indeed, it is less difficult and conservative to deal with a minimization problem than an equality constraint such as expressed by the structural conditions of the previous section.

\subsection{Control law design}

At each time instant $k$, the objective is to minimize the following criterion which is the norm of the tracking error:

$$
\Phi_{k}\left(u_{k}\right)=\left\|B_{k} u_{k}-x_{r, k+1}+A_{k} x_{k}\right\|_{W}^{2}
$$

where $W$ is a positive definite weighting matrix chosen accordingly to the state components for which some specific tracking is desired.

The control tracking law is then given by:

$$
u_{k}=\left(B_{k}^{T} W B_{k}\right)^{-1} B_{k}^{T} W\left(x_{r, k+1}-A_{k} x_{k}\right)
$$

where the matrices $A_{k}$ and $B_{k}$ have been already defined in (2).

Remark 2 When the premise variables $\xi_{k}$ depend on the control as explained previously (remark 1), the same procedure goes for the control law (19) with the iterative resolution, it becomes for $j=0, \ldots, N$ :

$$
u_{k}^{(j+1)}=\left(\left(B_{k}^{(j)}\right)^{T} W B_{k}^{(j)}\right)^{-1}\left(B_{k}^{(j)}\right)^{T} W\left(x_{r, k+1}-A_{k}^{(j)} x_{k}\right)
$$

with $B_{k}^{(j)}=\sum_{i=1}^{r} \mu_{i, k}\left(u_{k}^{(j)}\right) B_{i}, A_{k}^{(j)}=\sum_{i=1}^{r} \mu_{i, k}\left(u_{k}^{(j)}\right) A_{i}$.

The proposed control tracking law $u_{k}$ (19) (i.e. (20) for input dependent premise variables) aims to ensure the tracking of $x_{r, k+1}$ by $x_{k+1}$ at each time instant $k$. Since the controller has no ability to anticipate future events, it can not take control actions accordingly and the tracking performances may degrade. Based on this statement, the MPC tracking is introduced for the T-S models. In fact, the main advantage of MPC is to allow the current time slot to be optimized, while keeping future time slot in account. This is achieved by optimizing a finite time horizon, but only implementing the current time slot.

As for linear and nonlinear model reference tracking control, the MPC requires iterative solution and aims to ensure that the tracking error is minimized on a finite sliding horizon. Roughly speaking, the procedure for T-S models is the same as for the conventional MPC. However, some difficulties occur when the premise variables depend on the control.

\subsection{Premise variables independent of the input}

Considering a finite horizon of $p+1$ steps, using the state equation

$$
x_{k+1}=A_{k} x_{k}+B_{k} u_{k}
$$

it follows the state expression at time $k+p+1$

$$
\begin{aligned}
x_{k+p+1} & =A_{k+p} x_{k+p}+B_{k+p} u_{k+p} \\
& =\Phi_{k+p, k} x_{k}+\sum_{l=k}^{k+p-1} \Phi_{k+p, l+1} B_{l} u_{l}
\end{aligned}
$$

where

$$
\Phi_{k, l}=\left\{\begin{array}{l}
A_{k-1} \ldots A_{l} \text { if } k>l \geq 0 \\
I \text { if } k=l
\end{array}\right.
$$

with $A_{k}$ and $B_{k}$ defined by (2).

Gathering the states on the time horizon $[k+1: k+p+$ 1], let us note:

$\bar{x}_{k+1, p}=\left[\begin{array}{c}x_{k+1} \\ x_{k+2} \\ \vdots \\ x_{k+p+1}\end{array}\right], \bar{u}_{k, p}=\left[\begin{array}{c}u_{k} \\ u_{k+1} \\ \vdots \\ u_{k+p}\end{array}\right], \mathscr{A}_{k, p}=\left[\begin{array}{c}A_{k} \\ A_{k+1} A_{k} \\ \vdots \\ p \\ \prod_{i=0}^{p} A_{k+p-i}\end{array}\right]$

$\mathscr{B}_{k, p}=\left[\begin{array}{cccc}B_{k} & 0 & \ldots & 0 \\ A_{k+1} B_{k} & B_{k+1} & \ldots & 0 \\ \vdots & \vdots & \ddots & \vdots \\ \prod_{i=0}^{p-1} A_{k+p-i} B_{k} & \prod_{i=0}^{p-2} A_{k+p-i} B_{k+1} & \ldots & B_{k+p}\end{array}\right]$

Using (22) and (24), the state $\bar{x}$ is written as:

$$
\bar{x}_{k+1, p}=\mathscr{A}_{k, p} x_{k}+\mathscr{B}_{k, p} \bar{u}_{k, p}, \quad \bar{x}_{k, p} \in R^{n(p+1)}
$$

To ensure the reference model tracking on the time horizon $[k+1: k+p+1]$, the control $\bar{u}_{k, p}$ is ajusted in order to minimize the criterion:

$$
\Phi_{k, p}\left(\bar{u}_{k, p}\right)=\left\|\bar{x}_{r, k, p}-\mathscr{A}_{k, p} x_{k}-\mathscr{B}_{k, p} \bar{u}_{k, p}\right\|_{W}^{2}
$$

with $\bar{x}_{r, k, p}=\left[\begin{array}{lll}x_{r, k+1} & \ldots & x_{r, k+p+1}\end{array}\right]^{T} \in R^{n(p+1)}$. This leads to:

$$
\bar{u}_{k, p}=\left(\mathscr{B}_{k, p}^{T} W \mathscr{B}_{k, p}\right)^{-1} \mathscr{B}_{k, p}^{T} W\left(\bar{x}_{r, k, p}-\mathscr{A}_{k, p} x_{k}\right)
$$

where the input at the step $k$ defined by:

$$
u_{k}=\left[\begin{array}{llll}
I_{n_{u}} & 0 & \ldots & 0
\end{array}\right] \bar{u}_{k, p}
$$

is then applied to the system. For the next step, the horizon is moved and the criterion $\Phi_{k+1, p}$ is optimized in order to obtain and apply the control $u_{k+1}$. As explained before, the control $\bar{u}_{k, p}$ (in particular $u_{k}$ ) is calculated while keeping future time slot in account, which explains the anticipative character of the MPC control. 


\subsection{Extension to premise variables dependent of the input}

Since the weighting functions of the matrices $\mathscr{A}_{k, p}$ and $\mathscr{B}_{k, p}$ (24) may depend on the control $\bar{u}_{k, p}$, instead of the analytical solution (27), the following iterative algorithm is proposed:

1. define a threshold $\delta$

2. for $j=0$, define $\bar{u}_{k, p}^{(j)}$ and $\bar{u}_{k, p}^{(j-1)}$

3. compute $\mathscr{A}_{k, p}^{(j)}$ and $\mathscr{B}_{k, p}^{(j)}$

4. while $\left\|\bar{u}_{k, p}^{(j)}-\bar{u}_{k, p}^{(j-1)}\right\|>\delta$

$\bar{u}_{k, p}^{(j+1)}=\left(\left(\mathscr{B}_{k, p}^{(j)}\right)^{T} W \mathscr{B}_{k, p}^{(j)}\right)^{-1}\left(\mathscr{B}_{k, p}^{(j)}\right)^{T} W\left(\bar{x}_{r}-\mathscr{A}_{k, p}^{(j)} x_{k}\right)$

$j \leftarrow j+1$, compute $\mathscr{A}_{k, p}^{(j+1)}$ and $\mathscr{B}_{k, p}^{(j+1)}$.

with:

$$
\left\{\begin{array}{c}
\mathscr{A}_{k, p}^{(j)}=\left[\begin{array}{c}
A_{k}^{(j)} \\
A_{k+1}^{(j)} A_{k}^{(j)} \\
\vdots \\
\prod_{i=0}^{p} A_{k+p-i}^{(j)}
\end{array}\right], A_{k}^{(j)}=\sum_{i=1}^{r} \mu_{i, k}\left(u^{(j)}(k)\right) A_{i} \\
\mathscr{B}_{k, p}^{(j)}=\left[\begin{array}{cccc}
B_{k}^{(j)} & 0 & \ldots & 0 \\
\vdots & B_{k+1}^{(j)} B_{k}^{(j)} & \ldots & 0 \\
\vdots & \ddots & \vdots \\
\prod_{i=0}^{p-1} A_{k+p-i}^{(j)} B_{k}^{(j)} & \prod_{i=0}^{p-2} A_{k+p-i}^{(j)} B_{k+1}^{(j)} & \ldots & B_{k+p}^{(j)}
\end{array}\right] \\
B_{k}^{(j)}=\sum_{i=1}^{r} \mu_{i, k}\left(u^{(j)}(k)\right) B_{i}
\end{array}\right.
$$

After the algorithm convergence, the control input at the step $k$ is defined by:

$$
u_{k}=\left[\begin{array}{llll}
I_{n_{u}} & 0 & \ldots & 0
\end{array}\right] \bar{u}_{k, p}
$$

and is applied to the system. For the next step, the horizon is moved and the criterion $\Phi_{k+1, p}$ is optimized in order to obtain $u_{k+1}$.

\section{Numerical example}

In order to illustrate the proposed approach, let us consider the following numerical example:

$$
\begin{aligned}
& A_{r}=\left(\begin{array}{ccccc}
0.2 & 0.5 & 0 & 0 & 0 \\
-0.2 & 0.19 & -0.1 & 0 & 0 \\
0 & -1 & 0.2 & 0 & 0 \\
0 & 0.2 & -0.2 & 0.9 & -0.2 \\
0.1 & 0.3 & 0 & 0 & 0.7
\end{array}\right) \\
& A_{1}=\left(\begin{array}{ccccc}
0.3 & 0.5 & 0 & 0 & 0.1 \\
-0.2 & 0.69 & -0.1 & 0 & 0 \\
0 & -1.1 & 0.5 & 0 & 0.1 \\
-0.51 & -0.1 & 0 & 0.9 & -0.2 \\
0.1 & 0.3 & 0 & 0 & 1
\end{array}\right) \\
& A_{2}=\left(\begin{array}{ccccc}
0.6 & 0.5 & 0 & 0 & 0 \\
-0.2 & 0.39 & -0.1 & 0 & 0 \\
0 & -1 & 0.5 & 0 & 0 \\
0 & 0.2 & -0.2 & 1 & -0.2 \\
0.1 & 0.3 & 0 & 0 & 0.8
\end{array}\right) \\
& B_{1}=\left(\begin{array}{cc}
-0.2 & 0.3 \\
0.4 & 0.5 \\
0.3 & 1.3 \\
0.55 & 0.45 \\
-0.7 & -0.3
\end{array}\right), B_{2}=\left(\begin{array}{cc}
-0.8 & -0.3 \\
-0.2 & -0.1 \\
-0.3 & 0.7 \\
-0.05 & -0.15 \\
-1.3 & -0.9
\end{array}\right) \\
& B_{r}=\left(\begin{array}{cc}
-0.5 & 0 \\
0.1 & 0.2 \\
0 & 1 \\
0.25 & 0.15 \\
-1 & -0.6
\end{array}\right)
\end{aligned}
$$

The weighting functions are input dependent and given by:

$$
\left\{\begin{array}{l}
\mu_{1, k}=\frac{1+2 \tanh \left(u_{1, k}\right)}{2} \\
\mu_{2, k}=1-\mu_{1, k}
\end{array}\right.
$$

One can verify that the exact tracking conditions (8) are not fulfilled. For this reason, the MPC is performed for three steps forward $(p=2)$ with iterative resolution (30).

First, the objective is to ensure a good tracking of the fourth and fifth state components. Consequently, the weighting matrix is chosen as $W=\operatorname{diag}(1,1,1,2,2)$, implying a relaxation of the tracking of the first three state components. The system and reference model states are depicted in figure 3 (respectively noted $x_{i}$ 
and $\left.x_{i r}, i=1, \ldots, 5\right)$. In figure 4 , the control inputs $u_{r, k}$ and $u_{k}$ are represented. From the depicted figures, one can see that the control tracking is efficient (especially for the fourth and fifth states) although the structural conditions are not fulfilled.

Secondly, if the main objective is an accurate tracking

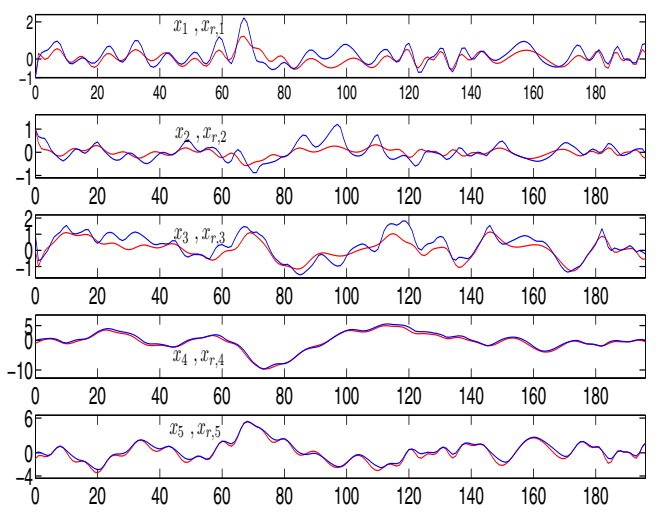

Figure 3. System and reference model states for $W=\operatorname{diag}(1,1,1,2,2)$
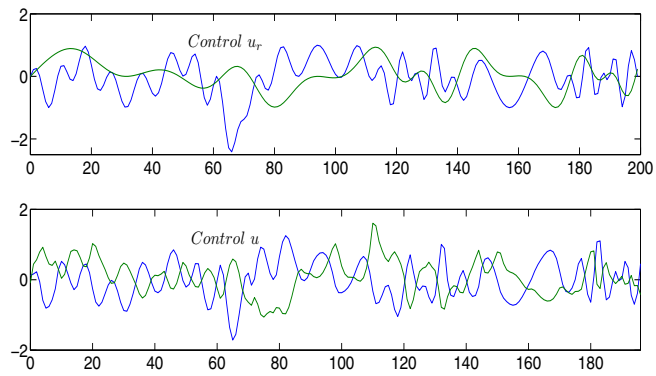

Figure 4. Control inputs $u_{r}(k)$ and $u(k)$

of $x_{3 r}$ by $x_{3}$, one should set the weighting matrix as $W=\operatorname{diag}(1,1,2,1,1)$. The obtained results are displayed in figure 5 for the system and model reference states and in figure 6 for the control inputs $u_{r, k}$ and $u_{k}$.

One can observe that for this case, the third state tracking has been improved when the fourth and fifth states tracking have been slightly deteriorated.

In order to quantify the improvement due to the predictive control, let us consider the following criterion:

$$
\phi_{i}=\sum_{k=0}^{N}\left|x_{r, k, i}-x_{k, i}\right|
$$

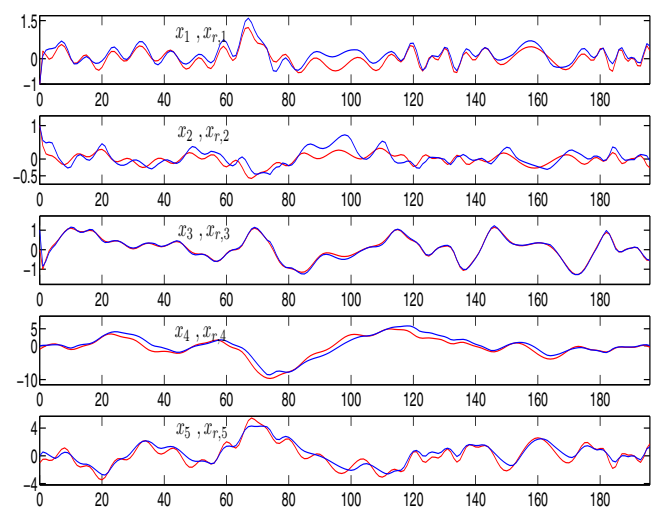

Figure 5. System and reference model states for $W=\operatorname{diag}(1,1,2,1,1)$
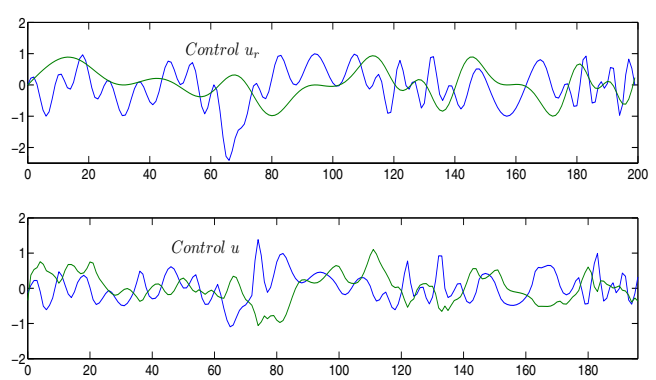

Figure 6. Control inputs $u_{r}(k)$ and $u(k)$

where $i$ the component number of a vector $(i=1, \ldots, 5)$, $N$ is the simulation horizon and $x_{k, i}$ is obtained from non predictive control (16).

$\phi_{i p}$ is analogously defined with $x_{k, i}$ obtained with MPC:

$$
\phi_{i p}=\sum_{k=0}^{N}\left|x_{r, k, i}-x_{k, i}^{p}\right|
$$

Finally, the performance gain $\tau_{i}$ due to MPC is obtained from

$$
\tau_{i}=100 \frac{\phi_{i}-\phi_{i p}}{\phi_{i}}
$$

For the considered example $(W=\operatorname{diag}(1,1,1,2,2))$, we obtain the following improvement (for each state): $\tau_{1}=2.54 \%, \tau_{2}=12.99 \%, \tau_{3}=5.21 \%, \tau_{4}=64.72 \%$, and $\tau_{5}=14.56 \%$.

In order to highlight the influence of the time horizon length $p+1$ on the tracking performances, the improvement of $\phi_{i}$, namely $\tau_{i}$, is computed for time horizon characterized by $p \in\{1,2,3,4\}$. The results are gathered in table I.

One can conclude, for the presented example, that a 


\begin{tabular}{|c|c|c|c|c|}
\hline & $p=1$ & $p=2$ & $p=3$ & $p=4$ \\
\hline$\tau_{1}$ & $1.76 \%$ & $2.54 \%$ & $8.99 \%$ & $14.02 \%$ \\
\hline$\tau_{2}$ & $4.80 \%$ & $12.99 \%$ & $22.28 \%$ & $28.85 \%$ \\
\hline$\tau_{3}$ & $3.81 \%$ & $5.21 \%$ & $6.21 \%$ & $7.67 \%$ \\
\hline$\tau_{4}$ & $46.90 \%$ & $64.72 \%$ & $77.52 \%$ & $81.1 \%$ \\
\hline$\tau_{5}$ & $10.62 \%$ & $14.56 \%$ & $13.64 \%$ & $14.81 \%$ \\
\hline
\end{tabular}

Table 1.

horizon of length $p=4$ gives the best results.

It is also important to highlight that depending on the dynamic characteristic of the reference on a time horizon, a too short, as well as a too long horizon may not give the best expected results. A compromise is then needed. To quantify the best horizon length, a comparative study as the one presented may be a good solution.

\section{Conclusion}

In this paper, the tracking objective for nonlinear T$\mathrm{S}$ model was considered. Structural conditions for perfect tracking were established, as well as the quadratic optimal control. A Model Predictive Control for the T$\mathrm{S}$ case with finite time horizon was also developed and the prediction influence was highlighted via a numerical example.

A first perspective for the present work is to generalize the matching conditions and the structure proposed in (10) for a general control structure law and establish the relation between the reference model $\left(A_{r}, B_{r}\right)$ and the system matrices $\left(A_{i}, B_{i}\right)$. During the study, a strong correlation between the time horizon length and the model reference dynamics was pointed, in fact, a second interesting perspective will be to present a choice criterion that optimizes the time horizon length according to the model reference dynamics.

\section{References}

[1] T. Takagi and M. Sugeno, "Fuzzy identification of systems and its applications to modeling and control," IEEE Transactions on Systems, Man and Cybernetics, vol. 15, no. 1, pp. 116-132, 1985.

[2] K. Tanaka and H. Wang, Fuzzy Control Systems Design and Analysis: A Linear Matrix Inequality Approach. John Wiley \& Sons, Inc., 2001.

[3] T. Taniguchi, K. Tanaka, K. Yumafuji, and H. Wang, "A new PDC fuzzy reference models," in IEEE International Fuzzy Systems Conference, Seoul, South Korea, 1999.
[4] C.-S. Tseng, B.-S. Chen, and H. Uang, "Fuzzy tracking control design for nonlinear dynamic systems via TS fuzzy model," IEEE Transactions on Fuzzy Systems, vol. 9, no. 3, pp. 381-392, 2001.

[5] B. Mansouri, N. Manamanni, K. Guelton, A. Kruszewski, and T. Guerra, "Output feedback LMI tracking control conditions with $H_{\infty}$ criterion for uncertain and disturbed T-S models," Information Sciences, vol. 179, no. 4, pp. 446-457, 2009.

[6] C. Nie and R. Patton, "Fault estimation and MRC-based active FTC," in $18^{\text {th }}$ IFAC World Congress, Milano, Italy, 2011.

[7] E. L. Allgöwer and K. Georg, "Simplicial and continuation methods for approximating fixed points and solutions to systems of equations," Society for Industrial and Applied Mathematics Revue, vol. 22, pp. 28-85, 1980.

[8] O. Axelsson, Iterative Solution Methods, C. U. Press, Ed. Cambridge, Australia: Cambridge University Press, 1994.

[9] C. T. Kelley, Iterative Methods for Linear and Nonlinear Equations. Philadelphia, Pennsylvanie, USA: Society for Industrial and Applied Mathematics, 1995. 